\title{
Enhanced dispersion and properties of a two-component epoxy nanocomposite using surface modified cellulose nanocrystals
}

Authors: Shane X. Peng ${ }^{1}$, Shikha Shrestha ${ }^{1}$, Youngman Yoo ${ }^{1}$, and Jeffrey P. Youngblood ${ }^{*}, 1$

\begin{abstract}
s
Epoxy nanocomposites reinforced with acetyl, hexanoyl, and dodecanoyl surface modified Cellulose Nanocrystals (CNC) were fabricated. Surface modified CNCs were dispersed in epoxy resin with a co-solvent. After complete removal of the co-solvent, a typical two component epoxy system was achieved where hardener was then added to cure the resin. Acetyl grafted CNC (CNC_a) and hexanoyl grafted CNC (CNC_h) both had good dispersion in cured epoxy as observed visually and through electron microscopy. Tensile modulus, tensile strength, and work of fracture of epoxy were improved with the addition of CNC_a and CNC_h. CNC_a reinforced epoxy had the highest increases in mechanical properties. Dodecanoyl grafted CNC (CNC_d) led to aggregation in the epoxy which led to insignificant changes in mechanical properties. The addition of $\mathrm{CNC}_{-}$a increased glass transition temperature $\left(\mathrm{T}_{\mathrm{g}}\right)$ of epoxy while CNC_h and CNC_d led to $\mathrm{T}_{\mathrm{g}}$ depression.
\end{abstract}

Keywords: Nanocomposite, Cellulose Nanocrystals, Epoxy, surface modification, mechanical properties 


\section{Introduction}

Cellulose nanocrystals (CNCs) belongs to a class of emerging cellulose based sustainable nanomaterials derived from biomass.[1-4] CNCs are whisker-shaped cellulose nanoparticles with high aspect ratio (3-10nm wide and 50 to 500nm long).[2] They are typically produced via acid hydrolysis of cellulose-rich source materials such as wood pulp.[5] Barring residual impurities from the production process, $\mathrm{CNC}$ consists almost exclusively of cellulose chains that are closely packed through intramolecular and intermolecular hydrogen bonding networks.[2,6] This ordered structure of CNC leads to high crystallinity,[7,8] good mechanical properties, $[9,10]$ good thermal properties, as well as anisotropy.[11,12] These intriguing properties along with the inherent sustainability of $\mathrm{CNC}$ have led to its utilization in a variety of different applications such as composites,[13-16] cements,[17,18] fibers,[19,20] electronics,[21-23] etc.

Among the different applications of $\mathrm{CNC}$, polymer nanocomposites utilizing $\mathrm{CNC}$ as reinforcing filler have drawn increasing attention. However, the inherent incompatibility between the hydrophilic $\mathrm{CNC}$ and the common hydrophobic polymer matrices remains a challenging issue during processing. Agglomeration of $\mathrm{CNC}$ in the polymer matrix usually leads to inferior mechanical and thermal properties of the overall nanocomposite. To prevent this, there are several ways to disperse CNC in polymer matrix. 1) Surfactant assisted dispersion: Either CNC or the polymer matrix is emulsified during processing to create dispersion.[24-28] The utilization of surfactant increased the complexity of the formulation of the final nanocomposite and the excess surfactant can cause issues. 2) Solvent assisted dispersion: Typically, CNCs are solvent exchanged from water to DMSO, DMF, or other co-solvent of the polymer matrix.[2934] The co-solvent is usually removed during casting or molding. This can lead to environmental emission and human exposure of volatile organic compounds (VOC). 3) Impregnation of 
preformed $\mathrm{CNC}$ : In this approach, $\mathrm{CNC}$ is freeze-dried in molds followed by the transfer/injection of polymer matrix resin.[35] The process requires a large scale and optimized lyophilization process to preserve shape integrity. 4) Surface modification of CNCs: The hydroxyl groups of cellulose can be functionalized via chemical reactions to change the surface chemistry of CNC.[36-38] This method can potentially increase the interfacial compatibility of CNC and the polymer matrix. Nevertheless, the type of surface modification needs to be carefully selected depending on the polymer matrix of interest.

In this study, a CNC/epoxy nanocomposite system is explored. Epoxy represents a class of thermoset polymer utilizing the cross-linking reaction of epoxide or oxirane moiety. Applications of epoxy can be found in many industries such as construction, aerospace, coating, electronic, and adhesives. Nanocomposites of epoxy utilizing various types of fillers can improve the mechanical, thermal, and electrical properties of neat epoxy resin.[39-42] There have been several previously reported CNC/epoxy nanocomposite studies utilizing some of the strategies stated above.[27,28,34,43-47] However, there has not been a study that explores the effect of CNC surface modification on the dispersion and final properties of epoxy nanocomposite. In addition, by improving dispersion, surface modification of $\mathrm{CNC}$ has the potential to eliminate the usage of solvent and dispersant during processing, thereby improving properties, reducing VOC emitted, and easing processing. In this work, we surface modified CNCs with aliphatic carboxylic acids. Acetyl, hexanoyl, and dodecanoyl grafted CNCs were generated using a previously established procedure. These surface modified CNCs were dispersed in epoxy resin to prepare a classic two component $(2 \mathrm{~K})$ epoxy system to be mixed with hardener and cured. The mechanical and thermal properties of the final epoxy nanocomposites were analyzed. The effects of the type of surface modification on the nanocomposite properties were compared. 


\section{Materials and methods}

\subsection{Materials}

Dimethyl Sulfoxide (DMSO, anhydrous 99.9\%), 1'1-Carbonyldiimidazole (CDI, reagent grade), Dodecanoic acid (98\%), Hexanoic acid (99.5\%), Acetic Anhydride (98\%), Pyridine (ACS reagent), Acetone, (ACS reagent), Dichloromethane (DCM, 99.5\%), DGEBA (Diglycidyl ether of bisphenol A, EEW=172-176), and Diethylenetriamine (DETA, AHEW=20.6) were purchased from Sigma Aldrich, St. Louis, MO, USA. Ethanol (200 proof) was purchased from VWR, West Chester, PA, USA. $11.8 \mathrm{wt} \% \mathrm{CNC}$ suspension in water was purchased from University of Maine and produced by USDA Forest Service-Forest Products Laboratory (FPL), Madison, WI, USA.[5] (Lot\# 2016-FPL-CNC-065)

\subsection{Surface modification of CNC}

CNCs were modified to graft acetyl, hexanoyl, and dodecanoyl side chain following a previous publication,[48] which can be referred to for detailed descriptions of the reaction procedures. The reaction schemes are illustrated in Figure 1. For Acetyl grafted CNC (CNC_a), $\mathrm{CNC}$ was solvent exchanged from water to acetone and then to pyridine. Acetic anhydride was then added to initiate the reaction following route 1 in Figure 1. For hexanoyl and dodecanoyl grafted $\mathrm{CNC}\left(\mathrm{CNC} \_\mathrm{h}\right.$ and $\left.\mathrm{CNC} \_\mathrm{d}\right), \mathrm{CNCs}$ were solvent exchanged from water to DMSO. Either dodecanoic acid or hexanoic acid was first mixed with CDI for 24 hours at $60{ }^{\circ} \mathrm{C}$. CNC/DMSO suspension was added to initiate the reaction following route 2 in Figure 1. All grafted CNCs 
were purified using a consecutive dispersion-centrifuge steps.[48] Water was used to purify CNC_a, while methanol and ethanol was used to purify CNC_h and CNC_d, respectively. After purification, all grafted $\mathrm{CNCs}$ were freeze-dried using a lyophilizer at $-80{ }^{\circ} \mathrm{C}$ (Labconco FreeZone Plus 4.5L). Degree of substitution of grafted CNCs were investigated with elemental analysis (Galbraith Laboratories, Knoxville, TN, USA).

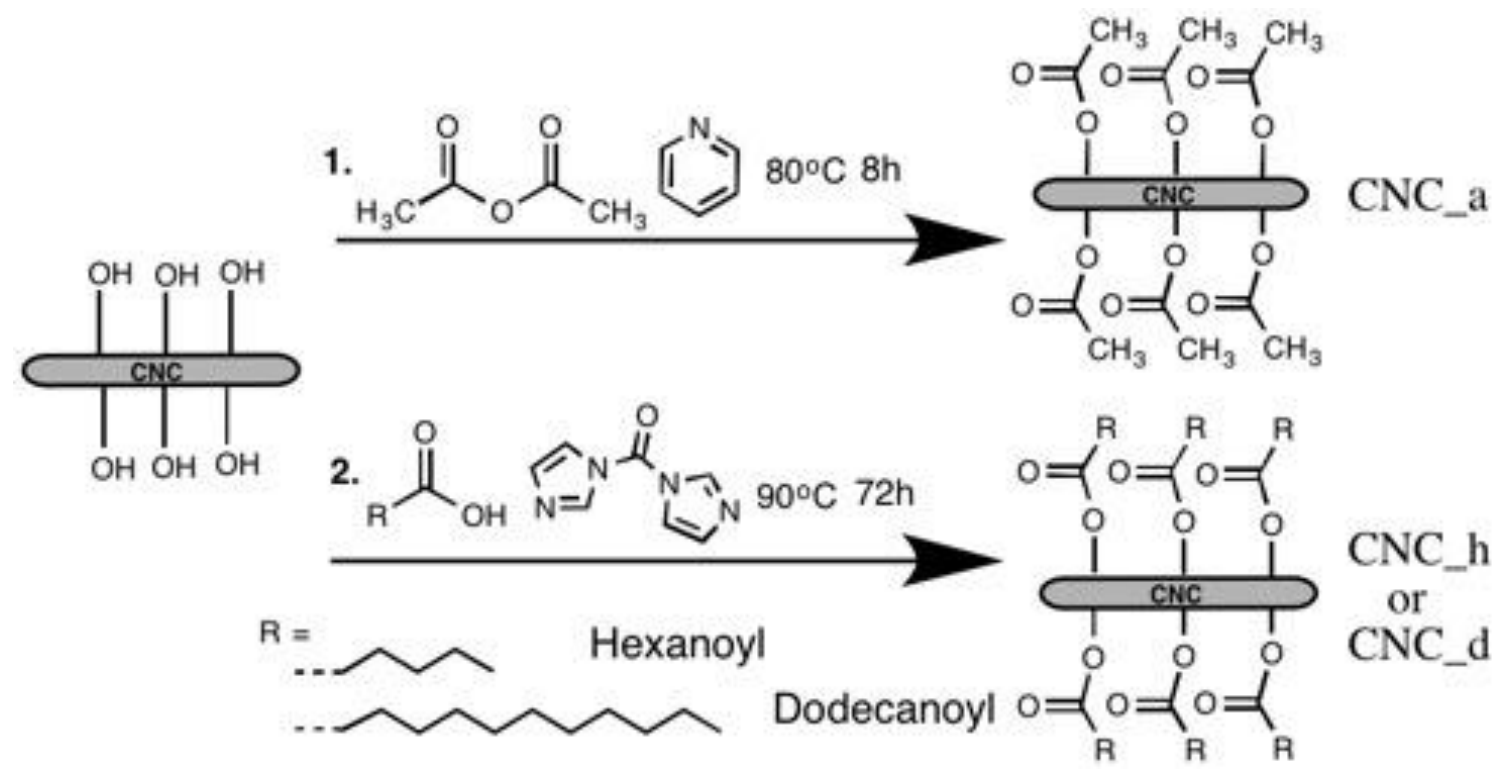

Figure 1. Reaction schemes of surface modification reactions of CNC

\subsection{Fabrication of grafted CNC/epoxy nanocomposite}

Freeze-dried grafted CNCs were first dispersed in acetone using an ultrasonicator (S250D, Branson Ultrasonics Corp., Danbury, CT, USA). For dodecanoyl grafted CNCs, DCM was used as solvent. DGEBA was then added to the grafted CNC suspension. The mixture was subsequently heated in a vacuum oven at $60{ }^{\circ} \mathrm{C}$ for 48 hours to remove solvent. After solvent removal, DETA was added under 1:1 amine to epoxide ratio. A planetary centrifugal mixer (DAC 400.1 FVZ, FlackTek Inc., Landrum, SC, USA) was used to combine DETA and grafted CNC/DGEBA mixture at $2500 \mathrm{rpm}$ for 2 minutes. Neat epoxy samples were fabricated using the 
sample procedure with similar amount of acetone added initially. All samples were cured at 60 ${ }^{\circ} \mathrm{C}$ for $1 \mathrm{~h}, 80{ }^{\circ} \mathrm{C}$ for $2 \mathrm{~h}$, and $125^{\circ} \mathrm{C}$ for $3 \mathrm{~h}$.

\subsection{UV-vis spectroscopy}

The CNC/epoxy samples were first manually ground and polished by using a LecoSpectrum System 1000 Grinder/Polisher. The samples were ground using coarser to finer abrasives (SiC paper), i.e., 320, 400, 600, 800, 1200 grit, along with continuously sprayed water as lubricant. The samples were subsequently polished using a diamond abrasive/paste (6 um) along with oil as lubricant.

After polishing, optical absorbance of individual epoxy specimens (thickness range from 0.2 to $0.8 \mathrm{~mm}$ ) was determined from UV-vis absorbance spectroscopy (SpectraMax Plus 384) in the wavelength range from 350 to $750 \mathrm{~nm}$, with a step of $10 \mathrm{~nm}$. Reproducibility was examined by conducting three measurements. The absorbance data is normalized to sample thickness.

\subsection{Polarized light microscopy}

Polished CNC/epoxy nanocomposite specimens were observed using a Carl Zeiss (Axio Observer A1) inverted microscope in transmission mode. The images were taken with the specimens between crossed polarizers using 20x magnification.

\subsection{Tensile Testing}

Uniaxial tensile testing was conducted using an MTS insight (MTS system Corp, Eden Prairie, MN, USA). The tensile specimens were laser cut following ASTM 638-10 Type IV dimensions proportionally decreased by two and half times. The experiments were conducted at 
$5 \mathrm{~mm} / \mathrm{min}$. At least ten replicates were tested for each type of specimen. Unpaired two-sample student t-tests were conducted to compare results with a threshold level set to 0.5 .

\subsection{Scanning Electron Microscopy (SEM)}

The fracture surface morphology of broken tensile specimens of neat epoxy and nanocomposites were inspected using a Phenom Pro Desktop SEM (Phenom-World B.V., Eindhoven, The Netherlands) at $10 \mathrm{kV}$. The surface of interest was mounted and sputter coated with gold.

2.8 Dynamic Mechanical Analysis (DMA)

DMA experiments (Q800 TA instruments, New Castle, DE, USA) were conducted on neat epoxy and different types of nanocomposites in single cantilever mode. The cured specimens were cut into $30 \mathrm{~mm}$ by $7.5 \mathrm{~mm}$ by $1.5 \mathrm{~mm}$ strips. The tests were conducted from room temperature to $220{ }^{\circ} \mathrm{C}$ with a heating rate $5{ }^{\circ} \mathrm{C} / \mathrm{min}$. The strain amplitude and frequency are set to $15 \mu \mathrm{m}$ and $1 \mathrm{~Hz}$, respectively.

\subsection{Differential Scanning Calorimetry (DSC)}

DSC measurements were conducted using TA Q100 (TA instruments, New Castle, DE, USA). Neat epoxy and nanocomposite specimens were cut and polished to ensure a flat surface. 15-20 mg of specimens were measured under nitrogen atmosphere using Tzero aluminum pans. The samples were first equilibrated to $180{ }^{\circ} \mathrm{C}$ and hold for 5 mins to remove thermal history, then cooled to $0{ }^{\circ} \mathrm{C}$ at $10{ }^{\circ} \mathrm{C} / \mathrm{min}$, and finally heated back up to $200{ }^{\circ} \mathrm{C}$ at $5{ }^{\circ} \mathrm{C} / \mathrm{min}$. The glass transition temperature $\left(T_{g}\right)$ were calculated from the final heating ramp. Three replicates of each types of specimens were measured. 


\section{Results and discussions}

\subsection{Surface modification of $\mathrm{CNC}$}

CNCs were surface modified following the procedure established in previous work.[48] A series of characterization experiments were conducted on surface modified CNCs as reported in previous work.[48] The detailed results can be found in the Supporting Information. Fourier Transform Infrared spectroscopy (FTIR) was used to qualitatively analyze the grafted side chains (Figure S1). The height of carbonyl stretch at $1740 \mathrm{~cm}^{-1}$ provides a rough indication of the extent of esterification reaction. Degree of substitution (DS) was calculated using results of elemental analysis of carbon, hydrogen, and oxygen content (Table S1). The preservation of crystallinity of CNC after each modification reaction was verified using X-ray diffraction (XRD) analysis (Figure S2). Deconvolution of XRD patterns were conducted to calculate the crystallinity of $\mathrm{CNC}$ after modification. The results in Table $\mathrm{S} 2$ indicate that no drastic change in crystallinity is observed, which is critical as high crystallinity of $\mathrm{CNC}$ correlates to its superior mechanical properties.[2]

\subsection{Dispersion of grafted CNC in epoxy}

Dispersion of grafted CNCs in epoxy resin after curing was evaluated visually. The sample pictures of epoxy specimens cured with different types of grafted CNC are shown in Figure 2. Each specimen is around $5 \mathrm{~mm}$ in thickness. As shown in Figure 2a, for CNC_a filled nanocomposites, the color of the specimen becomes darker and more yellow as weight content of CNC_a increased. However, the transparency of the specimen is not affected by the higher loading of filler. This indicates good dispersion of CNC_a in the epoxy matrix. When different types of grafted CNC are compared, CNC_a and CNC_h reinforced epoxy specimens have 
similar transparency as shown in Figure 2b. Despite having the same loading percentage as CNC_a and CNC_h, CNC_d specimen is cloudy and less transparent. This could be attributed to potential aggregations or phase separation in the specimen that leads to scattering of light. Since CNC_d has the longest aliphatic carbon side chains (dodecanoyl) and the most hydrophobic type of modification among all the grafted CNCs, dodecanoyl side chain could potentially be too hydrophobic for the epoxy matrix. We also anticipate that degree of substitution (DOS) of the surface modification plays a role, however a detailed study is beyond the scope here.

Optical absorbance of epoxy and nanocomposites films are illustrated in Figure 3. The trend found in Figure 3 followed the observation from Figure 2. The results indicated hexanoyl grafting (CNC_h) led to the best dispersion compared to other types of surface modifications. To further investigate dispersion of grafted $\mathrm{CNC}$ in epoxy, polarized light microscopy images were collected and illustrated in Figure 4. Birefringent domains observed in Figure 4b-4f represents CNC aggregations. As shown in Figure $4 b-4 d$, as concentration of grafted CNC increased, the birefringent domains increased. When CNC_a, CNC_h, and CNC_d are compared in Figure 4d4f, CNC_a and CNC_h had similar size and concentrations of birefringent domains, while CNC_d had larger domains indicating larger aggregation size. These results indicate the selection of the type of surface modification is important to obtain the optimal dispersion. To assist in this selection process, the teas plot based on Hanson's solubility parameter has been reported in previous studies.[48,49] However, being a multicomponent polymer system that undergoes cross-linking, cured epoxy has solubility parameters that depends on type of hardener used and curing conditions.[50] Therefore, individual dispersion tests of grafted CNC with respect to a particular system or formulation of epoxy may have to be conducted. To further 
correlate the extent of $\mathrm{CNC}$ dispersion to the performance of nanocomposite, mechanical properties of grafted CNC/epoxy nanocomposite are evaluated.

Overall, surface modification is a viable approach to improve dispersion of CNC into epoxy. Previous studies with epoxy often require water or solvents to be used as mixing media to ensure dispersion. The method introduced in this study is able to eliminate all solvents from the CNC/epoxy resin mixture before the epoxy resin is exposed to the hardener. This is advantageous as solvent can be captured during the epoxy resin production step and VOC exposure to the end-user can be prevented. In addition, by introducing CNC into the resin phase first without hardener, the epoxy formulation is not limited to a certain type of hardener or method of mixing, which can lead to more diversified applications. While some solvent is still used in the process to form the CNC/epoxy phase, the fact that it can be removed before mixing with hardener means that a traditional two component $(2 \mathrm{~K})$ epoxy formulation with no solvent on the consumer/application side. While solvent processing may have improved results here, all results presented are using this $2 \mathrm{~K}$ system and represent a consumer friendly system. 

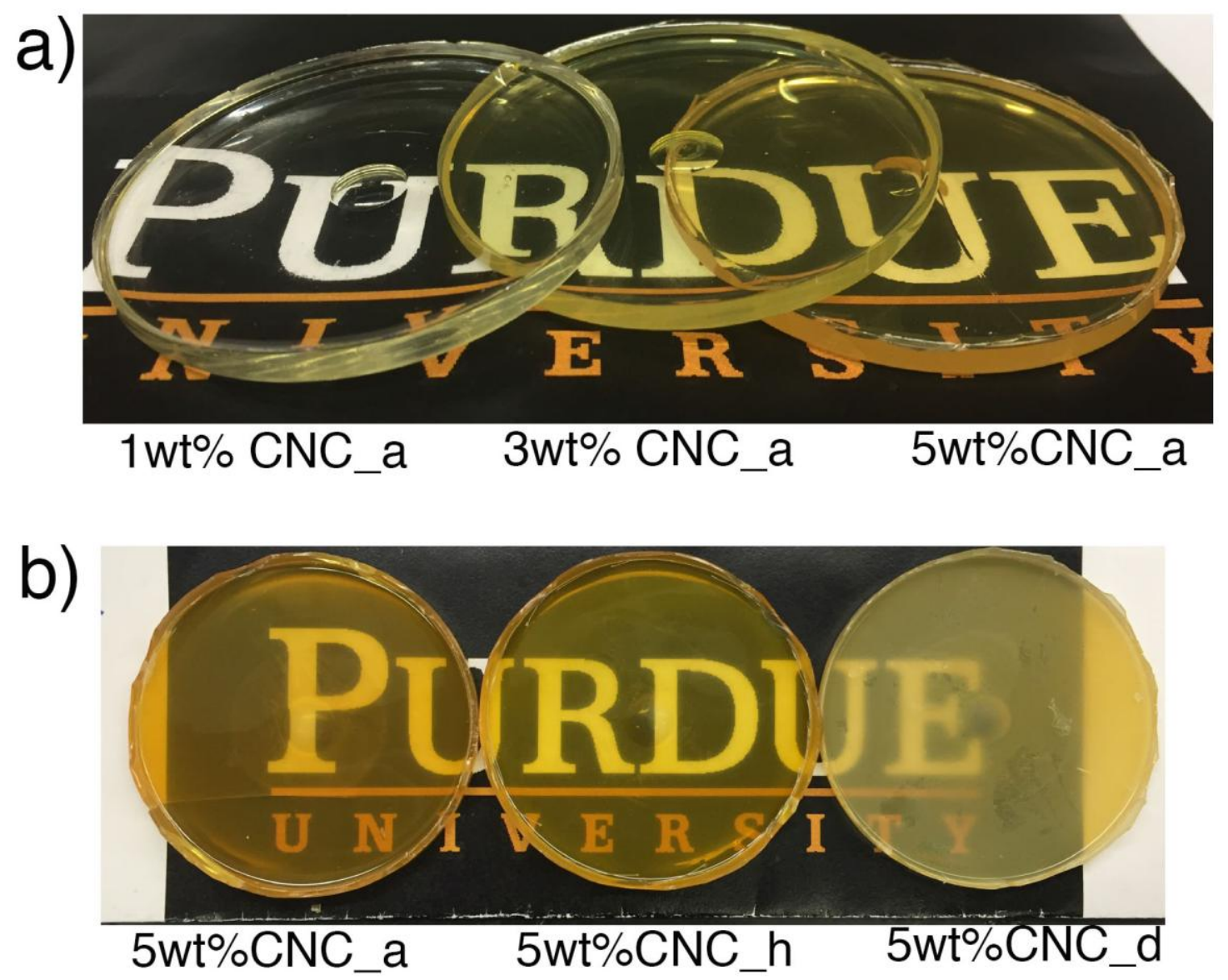

Figure 2. Sample pictures of epoxy nanocomposite with different types of grafted CNC

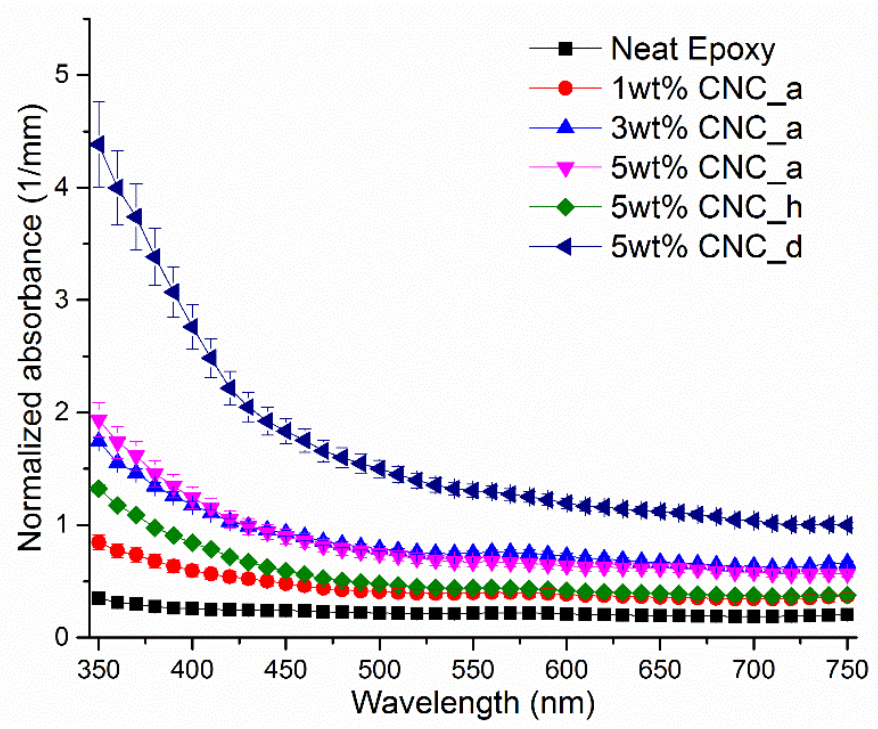

Figure 3. Optical absorbance of neat epoxy and epoxy nanocomposites with different types of grafted CNCs. 


\section{a)}
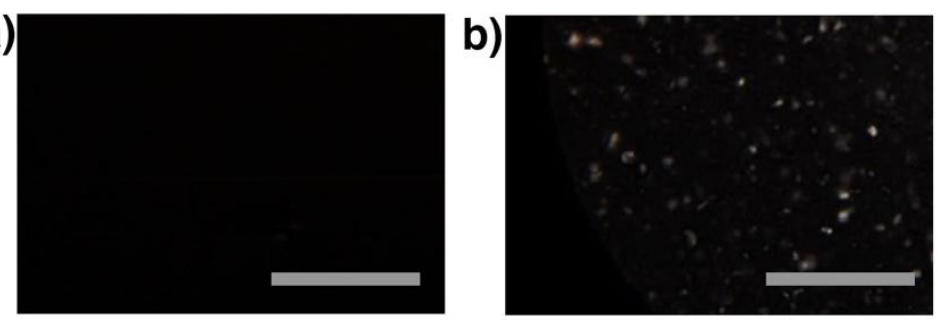

c)
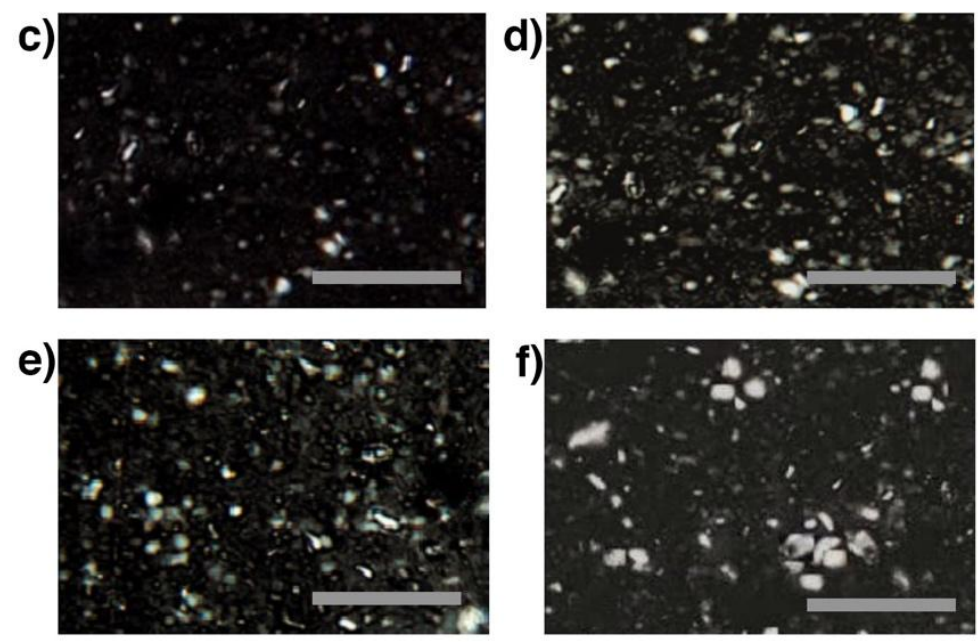

Figure 4. Polarized light microscopy images of neat epoxy (a) and epoxy nanocomposite with 1wt $\%$ CNC_a (b), 3wt \% CNC_a (c), 5wt \% CNC_a (d), 5wt\% CNC_h (e), and 5wt\% CNC_d (f). The scale bar is $100 \mu \mathrm{m}$.

\subsection{Mechanical properties of grafted CNC/epoxy nanocomposite}

The mechanical properties of grafted CNC/epoxy nanocomposites were evaluated via uniaxial tensile tests. Some of representative stress-strain curve are illustrated in Figure 5. The tensile properties are summarized in Figure 6. As shown in Figure 6a, CNC_a and CNC_h nanocomposites have similar trends of Young's modulus improvement with the CNC_a having seemingly slightly higher increases. However, the differences between CNC_a and CNC_h are statistically insignificant. For CNC_d, there is no statistically significant change in Young's modulus when $1 \mathrm{wt} \%$ and $5 \mathrm{wt} \%$ samples were compared with neat epoxy, while $3 \mathrm{wt} \%$ CNC_d loaded epoxy had statistically significant decrease. This could be due to the aggregation of 
CNC_d as observed in the sample pictures in Figure 6b. For ultimate tensile strength (UTS) shown in Figure 6b, CNC_a filled nanocomposite has the greatest increase among all the fillers. CNC_h specimens have an initial increase and reach a plateau. For CNC_d, there are statistically significant decreases in UTS for $1 \mathrm{wt} \%$ and $3 \mathrm{wt} \%$ specimens, while the change in $5 \mathrm{wt} \%$ specimens is insignificant when compared to neat epoxy. For strain at failure (SAF) shown in Figure 6c, the changes are statistically insignificant for most of grafted CNC samples when compared to neat epoxy samples, except for 3wt\% CNC_h, 5wt\% CNC_h, and 3wt\% CNC_d. This is likely due to the brittle nature of epoxy nature dominating the failure mechanism of the nanocomposite, as previous $\mathrm{CNC/epoxy} \mathrm{nanocomposite} \mathrm{studies} \mathrm{have} \mathrm{also} \mathrm{found} \mathrm{similar} \mathrm{strain} \mathrm{at}$ failure between filled epoxy and neat epoxy.[27,45] For work of fracture (WOF) in Figure 6d, the trend correlates to that of SAF data shown in Figure 6c. 3wt $\%$ and 5wt $\%$ CNC_a show statistically significant improvement of WOF comparing to neat epoxy, while $1 \mathrm{wt} \%$ CNC_d and 3wt\%CNC_d show decreases. For CNC_h samples, the changes are insignificant compared to neat epoxy. Lin et al.[51] and Zhang et al.[52] reported the tensile properties of poly(lactic acid) (PLA) and poly(butylene adipate-co-terephthalate) (PBAT) composite reinforced with acetylated CNCs, respectively. Both studies reported decreases in SAF as loading of acetylated CNC increased. In these studies, improvement in Young's modulus was also observed. Using hexanoyl and dodecanoyl grafted CNC, de Menezes et al. fabricated polyethylene (PE) nanocomposites.[37] In all of these studies, increases in modulus is observed, but decreases in SOF and WOF, indicating embrittlement of the polymer composite. However, as shown in Figure $6 \mathrm{~b}$ of this work, improvements in modulus, as well as UTS without loss of SOF is observed for CNC_a and CNC_h and an increase of WOF for CNC_a. These observations could be attributed to the better compatibly of CNC_a and CNC_h with the epoxy than PE. Initially, 
this may be rather surprising as aliphatic grafted $\mathrm{CNC}$ looks to have a more similar chemical structure to PE as opposed to amine cross linked bisphenol A (BPA) based epoxy resin. However, using solvency concepts such as Hansen's solubility parameter, it is seen that BPA epoxy is compatible with somewhat polar solvents such as acetone and MEK, while PE is more similar to solvents such as xylene. It has previously been reported that CNC_a is most compatible in ethanol, acetone, and terahydrofuran (THF), while CNC_d is too hydrophobic to disperse in polar solvents and $\mathrm{CNC}_{-} \mathrm{h}$ has dispersibility in between.[48] Thus, simply making CNCs as hydrophobic as possible is not sufficient, but rather the surface modification must be matched to the solvency of the polymer. As well, residual unreacted hydroxyl functionality improves interfacial bonding with epoxy, whereas would do little for PE.
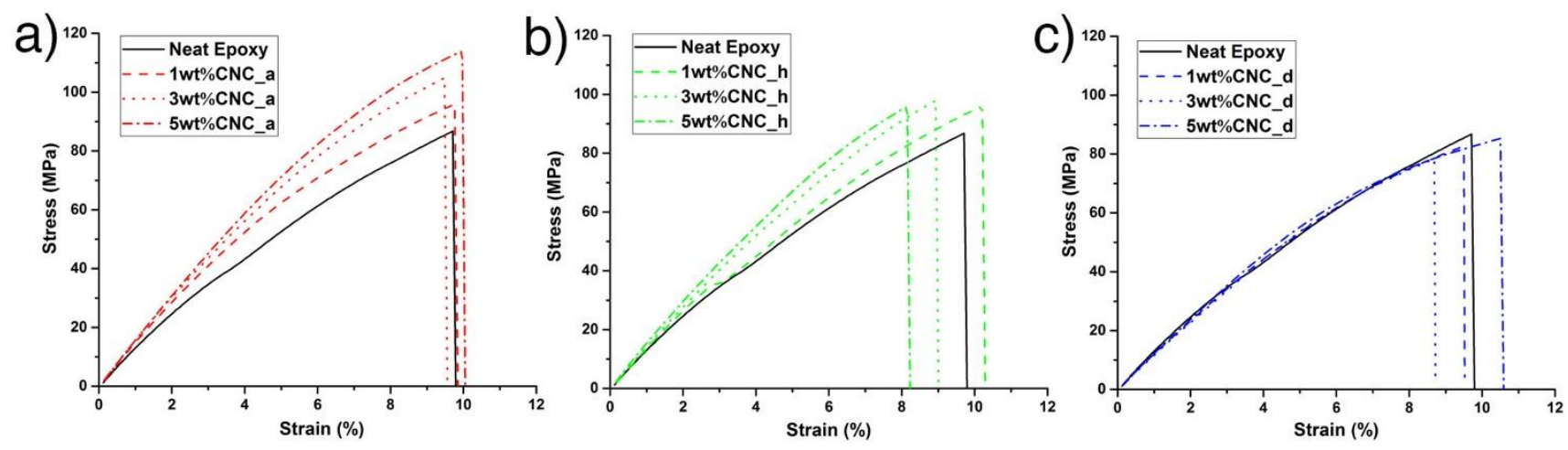

Figure 5. Stress-strain curves of neat epoxy and epoxy reinforced with a) CNC_a, b) CNC_h, and c) CNC_d. 
a)

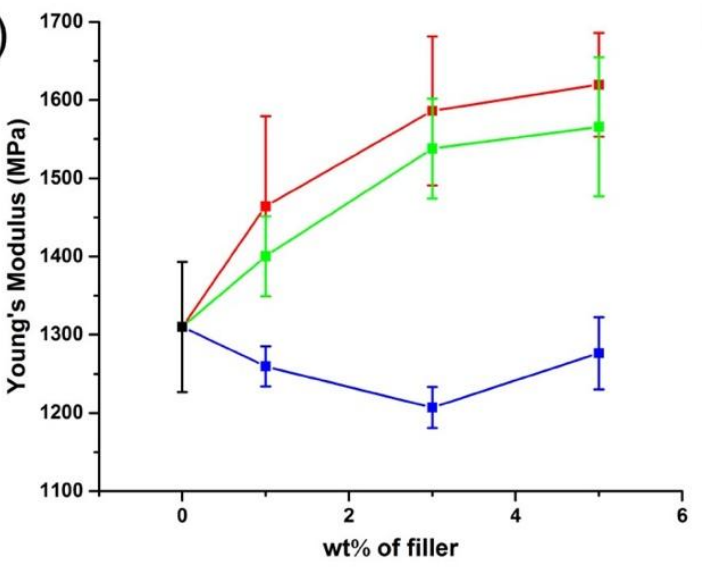

c)

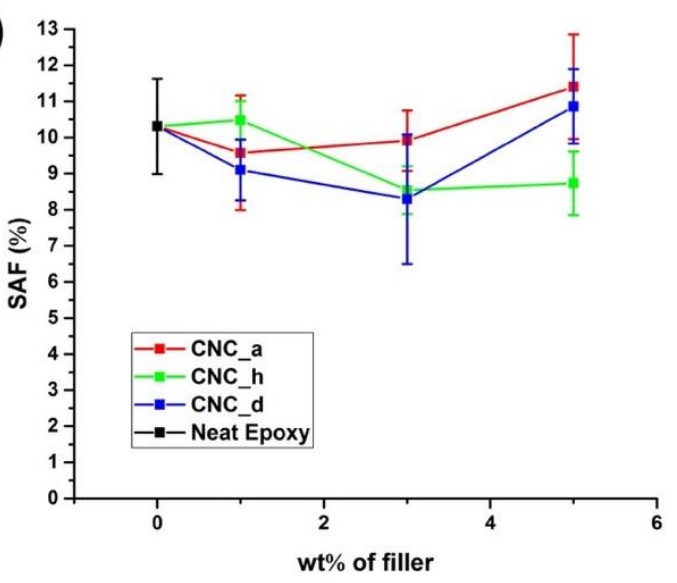

b)
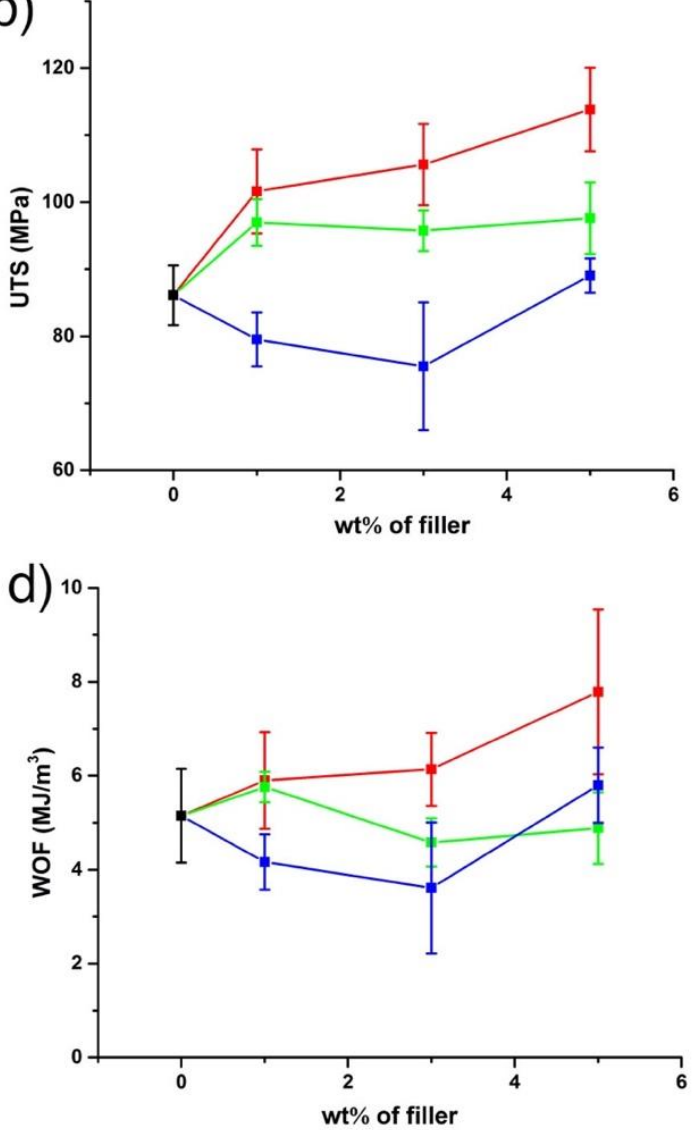

Figure 6. Tensile properties of different types of grafted CNC/epoxy nanocomposites: a) Young's modulus, b) Ultimate tensile strength (UTS), c) Strain at failure (SAF), and d) Work of fracture (WOF).

\subsection{Fracture surface morphology of grafted CNC/epoxy nanocomposite}

To further evaluate the effects of CNC surface grafting, fracture surface of the broken tensile specimens was observed using SEM. The SEM images of fracture surface are illustrated in Figure 7. Neat epoxy has a very smooth and homogenous fracture surface as shown in Figure 7a. The surface pattern indicates weak resistance to crack propagation that led to brittle failure as suggested by previous epoxy studies.[53,54] CNC_a and CNC_h filled epoxy had a similar pattern as neat epoxy at low magnification as shown in Figure $7 \mathrm{~b}$ and Figure 7c, respectively. 
For CNC_a filled nanocomposite, a rougher fracture surface with a smaller scale of roughness is observed at high magnification, indicating matrix shear yielding or local deformation similar to that of carbon nanofiber and alumina nanoparticle composite fracture surface.[53,54] In addition, no visible aggregation is observed for $\mathrm{CNC}_{-} \mathrm{a}$ and $\mathrm{CNC} \_\mathrm{h}$ filled specimens. Previous studies have reported white dots that represent individually dispersed CNC in the polymer matrix.[51,52] However, due to the resolution limit and the low contrast between CNC and epoxy matrix, individual CNC nanoparticles could not be observed. For the CNC_d filled nanocomposite shown in Figure 7d, an inhomogenous and rough fracture surface with large scale features is observed, possibly indicating aggregation of $\mathrm{CNC}$ rather than matrix shear yielding. The more magnified image also shows large white dots that potentially represent large agglomerations. Overall, the CNC_h filled nanocomposite has a fracture surface that is closest to neat epoxy and potentially has the best dispersion. This result correlated to that of optical absorbance data in Figure 3. However, a rough texture of CNC_a fracture surface could have suggested an alternative failure mechanism, where the presence of $\mathrm{CNC}$ potentially interrupted the crack propagation by randomly distributing the stress.[53,54] This mechanism could have led to the improvement in tensile strength and toughness observed in Figure 6b. 

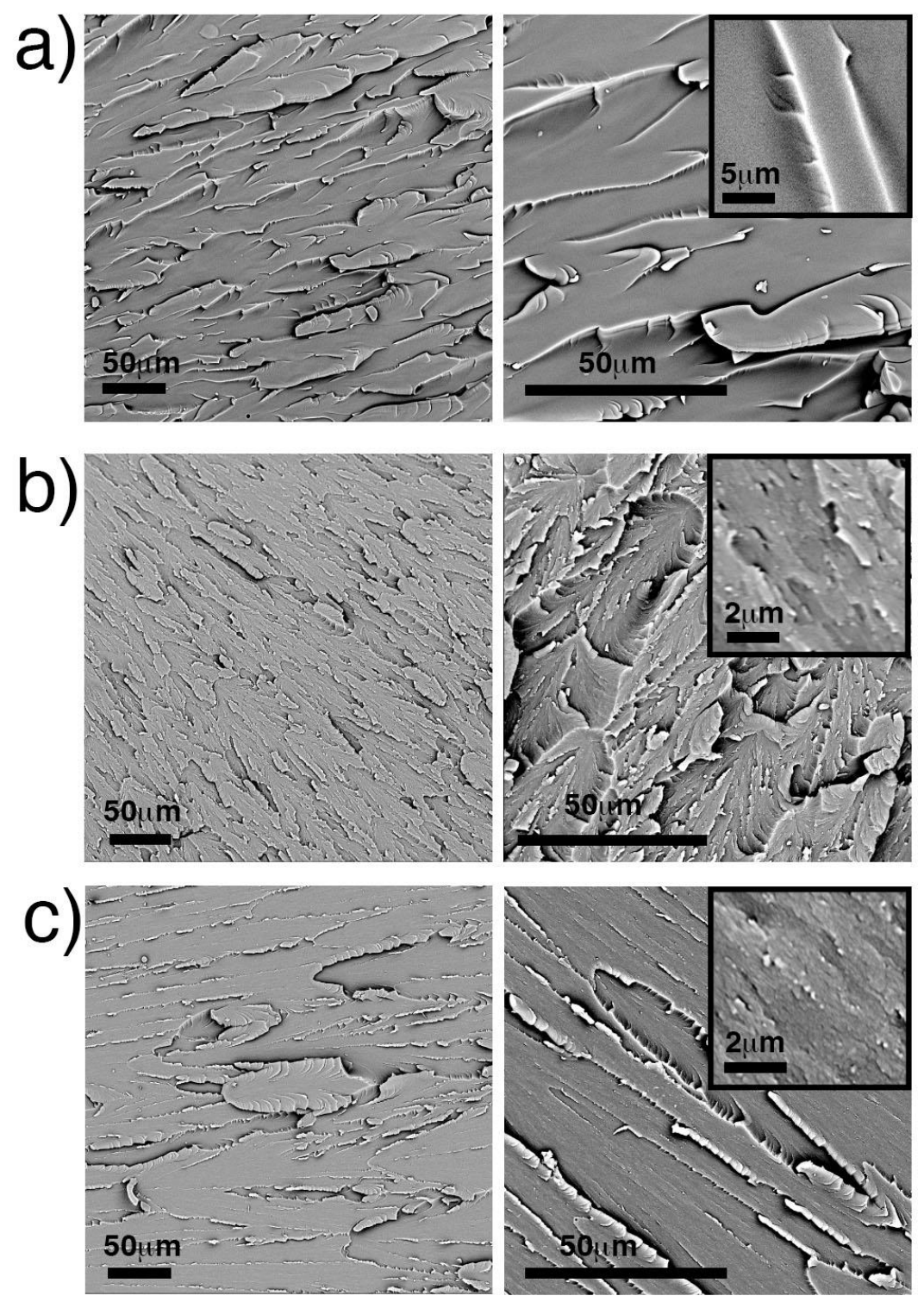

d)
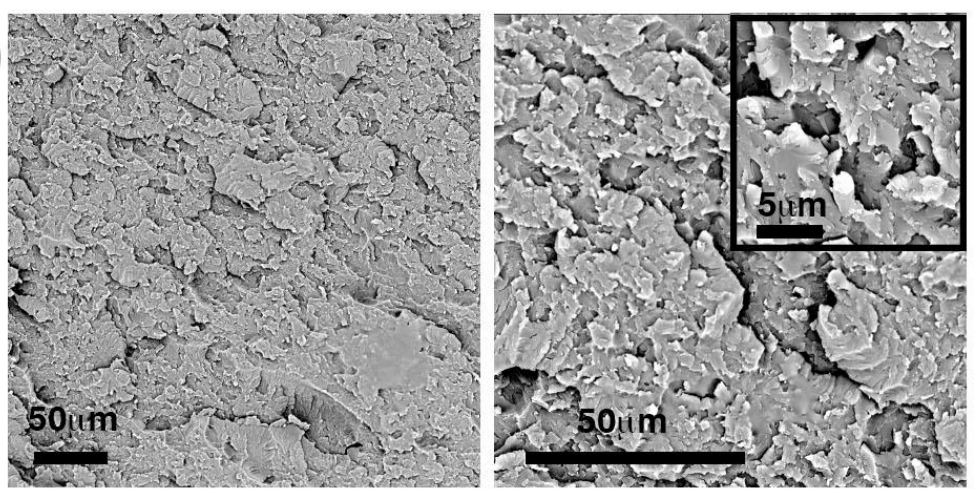

Figure 7. Fracture surface of broken tensile specimens of a) neat epoxy and epoxy with $5 \mathrm{wt} \%$ of

b) CNC_a, c) CNC_h, and d) CNC_d. 


\subsection{Thermal properties of grafted CNC/epoxy nanocomposite}

The thermomechanical properties of grafted CNC/epoxy nanocomposite were evaluated using DMA. The results are illustrated in Figure 8. For CNC_a nanocomposite, storage modulus $\left(E^{\prime}\right)$ above $\mathrm{T}_{\mathrm{g}}$ increased as the filler content increased as shown in Figure 8a. However, as Figure 8d suggested, at low CNC_a content, there is potentially a slight decrease in glass transition temperature $\left(\mathrm{T}_{\mathrm{g}}\right)$ when compared to neat epoxy as indicated by the Tan $\delta$ peak. The decrease in $\mathrm{T}_{\mathrm{g}}$ is more apparent for CNC_h and CNC_d epoxy nanocomposite. As suggested by Figure 8e and 8f, when the CNC_h and CNC_d content increase, the Tan $\delta$ maximum shift to lower temperature. Despite a decreased $\mathrm{T}_{\mathrm{g}}, E^{\prime}$ of $\mathrm{CNC} \_\mathrm{h}$ and $\mathrm{CNC} \_\mathrm{d}$ specimens are higher than neat epoxy above $T_{\mathrm{g}}$ as shown in Figure $8 \mathrm{~b}$ and $8 \mathrm{c}$. This indicates that despite surface modification, grafted CNC can still form strong interaction networks that facilitate stress transfer similar to that of unmodified CNCs reported previously, likely due to residual hydroxyls on the surface of the CNC.[27,28,34,43] Nevertheless, the depression of $\mathrm{T}_{\mathrm{g}}$ is uncommon for CNC filled nanocomposite. To further investigate the $\mathrm{T}_{\mathrm{g}}$ of epoxy and its nanocomposite, DSC studies were performed. 

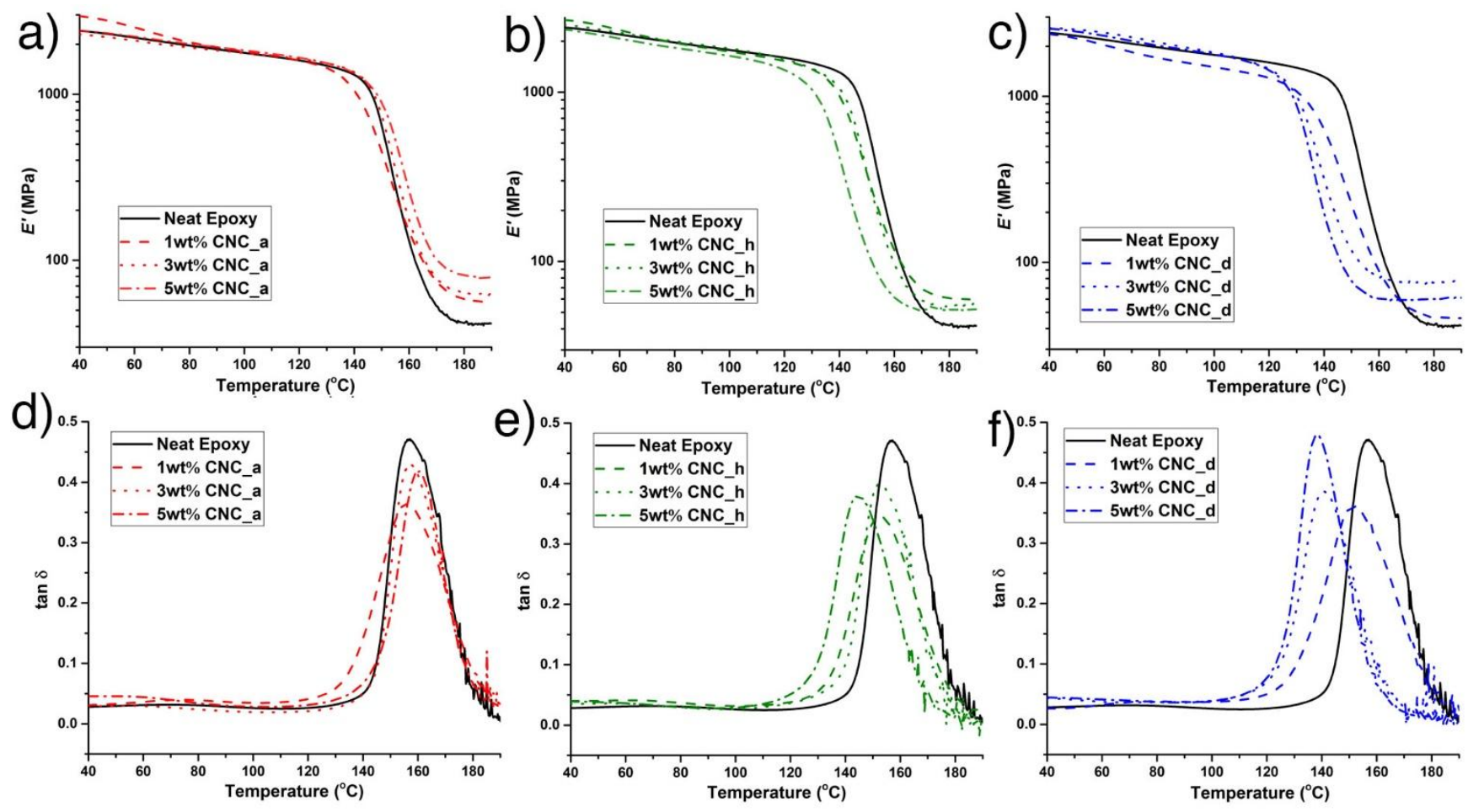

Figure 8. Thermomechanical properties of different types of grafted CNC/epoxy nanocomposite.

Storage modulus $\left(E^{\prime}\right)$ vs. Temperature are shown in Figure 8a-8c. Tan $\delta$ vs. Temperature are shown in Figure 8d-8f.

The $\mathrm{T}_{\mathrm{g}}$ of neat epoxy and grafted CNC/epoxy nanocomposites as measured by DSC is plotted in Figure 9. Similar trends in terms of $T_{g}$ changes are found as the Tan $\delta$ peaks suggested in Figure 8e to 8f. There are a few possible explanations for the $\mathrm{T}_{\mathrm{g}}$ depression in CNC_h and CNC_d specimens. One reason is the residual solvent from the fabrication steps. For this study, acetone or DCM was used as a co-solvent to disperse epoxy resin and grafted CNCs. As suggested by previous work, [14] the addition of acetone during the processing steps may lead to a reduced $\mathrm{T}_{\mathrm{g}}$ of cured epoxy. However, both CNC_a and CNC_h specimens were exposed to acetone during processing, only $\mathrm{CNC}_{-} \mathrm{h}$ specimens have a significant $\mathrm{T}_{\mathrm{g}}$ decrease. Another possibility is the aliphatic side chains of grafted $\mathrm{CNC}$ acted as a plasticizer, which led to $\mathrm{T}_{\mathrm{g}}$ 
depression. Esters of aliphatic acids are commonly used as plasticizer for vinyl polymers and rubber to increase proecssibility.[55] The main mechanism of action for aliphatic acid esters in vinyl polymer is to prevent intermolecular polar-polar interactions between neighboring matrix polymer chains by interacting with the polar sites on the matrix polymer themselves. A similar mechanism has been speculated in previous study on hexanoyl and dodecanoyl grafted $\mathrm{CNC} / \mathrm{PE}$ nanocomposite, where an additional higher relaxation temperature was generated in PE with addition of grafted CNC.[37] The author suggested potential interactions of grafted side chain and PE matrix led to this new relaxation. However, such interaction is not expected between grafted CNC and cured epoxy network. Yet, longer aliphatic side chains such as hexanoyl and dodecanoyl may introduce localized flexibility and create more free volume, which would lead to decreases in $T_{\mathrm{g}}$.

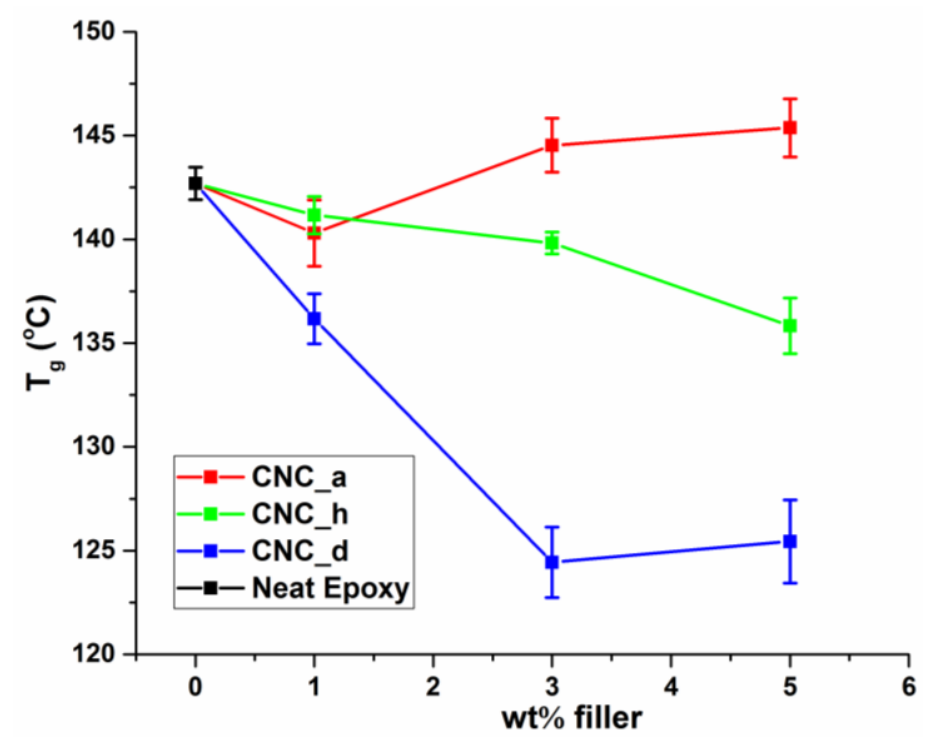

Figure 9. Glass transition temperature as measured by dynamic scanning calorimetry of cured neat epoxy and different types of grafted CNC/epoxy nanocomposite. 


\section{Conclusions}

In this study, epoxy nanocomposites with different types of surface modified CNCs were fabricated. CNCs were grafted with acetyl, hexanoyl, and dodecanoyl side chains via esterification reactions. The surface modified CNCs were dispersed in the epoxy phase and after processing a solventless $2 \mathrm{~K}$ epoxy system subsequent cured with DETA amine hardener was prepared. CNC_a and $\mathrm{CNC} \_\mathrm{h}$ had good dispersion in the cured epoxy matrix, while CNC_d led to agglomerations. $\mathrm{CNC} \_\mathrm{a}$ and $\mathrm{CNC} \_\mathrm{h}$ had positive reinforcement of epoxy matrix. CNC_a increased Young's Modulus up to 23\%, tensile strength up to 29\%, and work of fracture up to $56 \%$ with 5wt\% loading. CNC_d did have significant effects on the mechanical properties of epoxy resin. $\mathrm{T}_{\mathrm{g}}$ increased up to $5^{\circ} \mathrm{C}$ with $\mathrm{CNC} \_\mathrm{a}$ addition, but decreased with $\mathrm{CNC} \_\mathrm{h}$ and $\mathrm{CNC} \_\mathrm{d}$ addition. Overall, acetylation of CNC gave the best combination of mechanical and thermal property enhancement of epoxy likely due to it better matching the epoxy phase in terms of polarity.

\section{Supporting Information}

FTIR spectra of different types of surface modified CNCs, Table of elemental analysis results and degree of substation different types of surface modified CNCs, XRD diffractograms of different types of surface modified CNCs, Table of calculated degree of crystallinity of different types of surface modified CNCs 


\section{Acknowledgement}

This research was supported by the National Science Foundation IGERT sustainable electronic grant\#1144843-DGE and the Forest Products Laboratory under USDA grant\# 11-JV11111129-118

\section{References}

[1] C.S. Davis, D.L. Grolman, A. Karim, J.W. Gilman, What do we still need to understand to commercialize cellulose nanomaterials?, Green Mater. 3 (2015) 53-58. doi:10.1680/jgrma.15.00013.

[2] R.J. Moon, A. Martini, J. Nairn, J. Simonsen, J. Youngblood, Cellulose nanomaterials review: structure, properties and nanocomposites., Chem. Soc. Rev. 40 (2011) 3941-94. doi:10.1039/c0cs00108b.

[3] F.G. Torres, O.P. Troncoso, C. Torres, C.J. Grande, Advances in Natural Polymers, Springer Berlin Heidelberg, Berlin, Heidelberg, 2013. doi:10.1007/978-3-642-20940-6.

[4] O. Faruk, A.K. Bledzki, H.-P. Fink, M. Sain, Progress Report on Natural Fiber Reinforced Composites, Macromol. Mater. Eng. 299 (2014) 9-26. doi:10.1002/mame.201300008.

[5] R.S. Reiner, A.W. Rudie, Process Scale-Up of Cellulose Nanocrystal Production to $25 \mathrm{~kg}$ per Batch at the Forest Products Laboratory, in: M.T. Postek, R.J. Moon, A.W. Rudie, M.A. Bilodeau (Eds.), Prod. Appl. Cellul. Nanomater., TAPPI Press, Peachtree Corners, GA, 2013: pp. 21-24. 
[6] A.C. O'Sullivan, Cellulose: the structure slowly unravels, Cellulose. 4 (1997) 173-207. doi:10.1023/A:1018431705579.

[7] S. Park, J.O. Baker, M.E. Himmel, P. a Parilla, D.K. Johnson, Cellulose crystallinity index: measurement techniques and their impact on interpreting cellulase performance, Biotechnol. Biofuels. 3 (2010) 10. doi:10.1186/1754-6834-3-10.

[8] S.H. Kim, C.M. Lee, K. Kafle, Characterization of crystalline cellulose in biomass: Basic principles, applications, and limitations of XRD, NMR, IR, Raman, and SFG, Korean J. Chem. Eng. 30 (2013) 2127-2141. doi:10.1007/s11814-013-0162-0.

[9] R.R. Lahiji, X. Xu, R. Reifenberger, A. Raman, A. Rudie, R.J. Moon, Atomic force microscopy characterization of cellulose nanocrystals., Langmuir. 26 (2010) 4480-4488. doi:10.1021/la903111j.

[10] R. Wagner, R.J. Moon, A. Raman, Mechanical properties of cellulose nanomaterials studied by contact resonance atomic force microscopy, Cellulose. 23 (2016) 1031-1041. doi:10.1007/s10570-016-0883-4.

[11] J.A. Diaz, Z. Ye, X. Wu, A.L. Moore, R.J. Moon, A. Martini, D.J. Boday, J.P. Youngblood, Thermal Conductivity in Nanostructured Films: From Single Cellulose Nanocrystals to Bulk Films, Biomacromolecules. 15 (2014) 4096-4101. doi:10.1021/bm501131a.

[12] J.A. Diaz, X. Wu, A. Martini, J.P. Youngblood, R.J. Moon, Thermal Expansion of SelfOrganized and Shear-Oriented Cellulose Nanocrystal Films, Biomacromolecules. 14 (2013) 2900-2908. doi:10.1021/bm400794e.

[13] C. Miao, W.Y. Hamad, Cellulose reinforced polymer composites and nanocomposites: a critical review, Cellulose. 20 (2013) 2221-2262. doi:10.1007/s10570-013-0007-3. 
[14] S.X. Peng, R.J. Moon, J.P. Youngblood, Design and characterization of cellulose nanocrystal-enhanced epoxy hardeners, Green Mater. 2 (2014) 193-205. doi:10.1680/gmat.14.00015.

[15] J.-C. Liu, D.J. Martin, R.J. Moon, J.P. Youngblood, Enhanced thermal stability of biomedical thermoplastic polyurethane with the addition of cellulose nanocrystals, J. Appl. Polym. Sci. 132 (2015) 41970. doi:10.1002/app.41970.

[16] J.-C. Liu, R.J. Moon, A. Rudie, J.P. Youngblood, Mechanical performance of cellulose nanofibril film-wood flake laminate, Holzforschung. 68 (2014) 283-290. doi:10.1515/hf2013-0071.

[17] Y. Cao, P. Zavattieri, J. Youngblood, R. Moon, J. Weiss, The relationship between cellulose nanocrystal dispersion and strength, Constr. Build. Mater. 119 (2016) 71-79. doi:10.1016/j.conbuildmat.2016.03.077.

[18] Y. Cao, P. Zavaterri, J. Youngblood, R. Moon, J. Weiss, The influence of cellulose nanocrystal additions on the performance of cement paste, Cem. Concr. Compos. 56 (2015) 73-83. doi:10.1016/j.cemconcomp.2014.11.008.

[19] S. Chen, G. Schueneman, R.B. Pipes, J. Youngblood, R.J. Moon, Effects of Crystal Orientation on Cellulose Nanocrystals-Cellulose Acetate Nanocomposite Fibers Prepared by Dry Spinning, Biomacromolecules. 15 (2014) 3827-3835. doi:10.1021/bm501161v.

[20] M. Ago, K. Okajima, J.E. Jakes, S. Park, O.J. Rojas, Lignin-Based Electrospun Nanofibers Reinforced with Cellulose Nanocrystals, Biomacromolecules. 13 (2012) 918926. doi:10.1021/bm201828g.

[21] Y. Zhou, C. Fuentes-Hernandez, T.M. Khan, J.-C. Liu, J. Hsu, J.W. Shim, A. Dindar, J.P. Youngblood, R.J. Moon, B. Kippelen, Recyclable organic solar cells on cellulose 
nanocrystal substrates, Sci. Rep. 3 (2013) 327-332. doi:10.1038/srep01536.

[22] Y. Zhou, T.M. Khan, J.C. Liu, C. Fuentes-Hernandez, J.W. Shim, E. Najafabadi, J.P. Youngblood, R.J. Moon, B. Kippelen, Efficient recyclable organic solar cells on cellulose nanocrystal substrates with a conducting polymer top electrode deposited by film-transfer lamination, Org. Electron. Physics, Mater. Appl. 15 (2014) 661-666.

doi:10.1016/j.orgel.2013.12.018.

[23] C.-Y. Wang, C. Fuentes-Hernandez, J.-C. Liu, A. Dindar, S. Choi, J.P. Youngblood, R.J. Moon, B. Kippelen, Stable Low-Voltage Operation Top-Gate Organic Field-Effect Transistors on Cellulose Nanocrystal Substrates, ACS Appl. Mater. Interfaces. 3 (2015) 150218115157002. doi:10.1021/am508723a.

[24] N.L. Garcia de Rodriguez, W. Thielemans, A. Dufresne, Sisal cellulose whiskers reinforced polyvinyl acetate nanocomposites, Cellulose. 13 (2006) 261-270. doi:10.1007/s10570-005-9039-7.

[25] M. Roohani, Y. Habibi, N.M. Belgacem, G. Ebrahim, A.N. Karimi, A. Dufresne, Cellulose whiskers reinforced polyvinyl alcohol copolymers nanocomposites, Eur. Polym. J. 44 (2008) 2489-2498. doi:10.1016/j.eurpolymj.2008.05.024.

[26] G. Wu, J. Chen, S. Huo, G. Liu, Z. Kong, Thermoset nanocomposites from twocomponent waterborne polyurethanes and cellulose whiskers, Carbohydr. Polym. 105 (2014) 207-213. doi:10.1016/j.carbpol.2014.01.095.

[27] S. Xu, N. Girouard, G. Schueneman, M.L. Shofner, J.C. Meredith, Mechanical and thermal properties of waterborne epoxy composites containing cellulose nanocrystals, Polymer (Guildf). 54 (2013) 6589-6598. doi:10.1016/j.polymer.2013.10.011.

[28] M.M. Ruiz, J.Y. Cavaille, A. Dufresne, C. Graillat, J.-F. Gerard, New waterborne epoxy 
coatings based on cellulose nanofillers., Macromol. Symp. 169 (2001) 211-222. doi:10.1002/1521-3900(200105)169:1<211::AID-MASY211>3.0.CO;2-H.

[29] A. Iwatake, M. Nogi, H. Yano, Cellulose nanofiber-reinforced polylactic acid, Compos. Sci. Technol. 68 (2008) 2103-2106. doi:10.1016/j.compscitech.2008.03.006.

[30] M. Kowalczyk, E. Piorkowska, P. Kulpinski, M. Pracella, Mechanical and thermal properties of PLA composites with cellulose nanofibers and standard size fibers, Compos. Part A Appl. Sci. Manuf. 42 (2011) 1509-1514. doi:10.1016/j.compositesa.2011.07.003.

[31] N.E. Marcovich, M.L. Auad, N.E. Bellesi, S.R. Nutt, M.I. Aranguren, Cellulose micro/nanocrystals reinforced polyurethane, J. Mater. Res. 21 (2006) 870-881. doi:10.1557/jmr.2006.0105.

[32] L. Rueda, A. Saralegui, B. Fernández D’Arlas, Q. Zhou, L.A. Berglund, M.A. Corcuera, I. Mondragon, A. Eceiza, Cellulose nanocrystals/polyurethane nanocomposites. Study from the viewpoint of microphase separated structure, Carbohydr. Polym. 92 (2013) 751-757. doi:10.1016/j.carbpol.2012.09.093.

[33] J. Pan, D.G. Li, Q.Y. Deng, Y.M. Wang, D.L. Lin, New Nanocomposite Based on the Cellulose Nanofibers and Epoxy Resin, Appl. Mech. Mater. 174-177 (2012) 761-766. doi:10.4028/www.scientific.net/AMM.174-177.761.

[34] L. Tang, C. Weder, Cellulose whisker/epoxy resin nanocomposites., ACS Appl. Mater. Interfaces. 2 (2010) 1073-80. doi:10.1021/am900830h.

[35] B. Barari, T.K. Ellingham, I.I. Ghamhia, K.M. Pillai, R. El-Hajjar, L.S. Turng, R. Sabo, Mechanical characterization of scalable cellulose nano-fiber based composites made using liquid composite molding process, Compos. Part B Eng. 84 (2016) 277-284. doi:10.1016/j.compositesb.2015.08.040. 
[36] A. Sdrobi, R.N. Darie, M. Totolin, G. Cazacu, C. Vasile, Low density polyethylene composites containing cellulose pulp fibers, Compos. Part B Eng. 43 (2012) 1873-1880. doi:10.1016/j.compositesb.2012.01.064.

[37] A. Junior de Menezes, G. Siqueira, A.A.S. Curvelo, A. Dufresne, Extrusion and characterization of functionalized cellulose whiskers reinforced polyethylene nanocomposites, Polymer (Guildf). 50 (2009) 4552-4563. doi:10.1016/j.polymer.2009.07.038.

[38] T. Lu, M. Jiang, Z. Jiang, D. Hui, Z. Wang, Z. Zhou, Effect of surface modification of bamboo cellulose fibers on mechanical properties of cellulose/epoxy composites, Compos. Part B Eng. 51 (2013) 28-34. doi:10.1016/j.compositesb.2013.02.031.

[39] J. Guo, J. Long, D. Ding, Q. Wang, Y. Shan, A. Umar, X. Zhang, B.L. Weeks, S. Wei, Z. Guo, Significantly enhanced mechanical and electrical properties of epoxy nanocomposites reinforced with low loading of polyaniline nanoparticles, RSC Adv. 6 (2016) 21187-21192. doi:10.1039/C5RA25210E.

[40] H.B. Gu, J. Guo, H.G. Wei, X.R. Yan, D.W. Ding, X. Zhang, Q.L. He, S. Tadakamalla, X.F. Wang, T.C. Ho, S.Y. Wei, Z.H. Guo, Transparent anhydride-cured epoxy nanocomposites reinforced with polyaniline stabilized nanosilica, J. Mater. Chem. C. 3 (2015) 8152-8165. doi:10.1039/c5tc01392e.

[41] H. Gu, C. Ma, J. Gu, J. Guo, X. Yan, J. Huang, Q. Zhang, Z. Guo, An Overview of Multifunctional Epoxy Nanocomposites, J. Mater. Chem. C. 4 (2016) 5890-5906. doi:10.1039/C6TC01210H.

[42] H. Gu, J. Guo, H. Wei, S. Guo, J. Liu, Y. Huang, M.A. Khan, X. Wang, D.P. Young, S. Wei, Z. Guo, Strengthened Magnetoresistive Epoxy Nanocomposite Papers Derived from 
Synergistic Nanomagnetite-Carbon Nanofiber Nanohybrids, Adv. Mater. 27 (2015) 62776282. doi:10.1002/adma.201501728.

[43] M. Matos Ruiz, J.Y. Cavaillé, A. Dufresne, J.F. Gérard, C. Graillat, Processing and characterization of new thermoset nanocomposites based on cellulose whiskers, Compos. Interfaces. 7 (2000) 117-131. doi:10.1163/156855400300184271.

[44] Y. Shimazaki, Y. Miyazaki, Y. Takezawa, M. Nogi, K. Abe, S. Ifuku, H. Yano, H. Chemical, Excellent Thermal Conductivitiy of Transparent Cellulose Nanofiber/Epoxy Resin Nanocomposites, Biomacromolecules. 8 (2007) 2976-2978.

[45] N. Girouard, G.T. Schueneman, M.L. Shofner, J.C. Meredith, Exploiting colloidal interfaces to increase dispersion, performance, and pot-life in cellulose nanocrystal/waterborne epoxy composites, Polymer (Guildf). 68 (2015) 111-121. doi:10.1016/j.polymer.2015.05.009.

[46] Z. Emami, Q. Meng, G. Pircheraghi, I. Manas-Zloczower, Use of surfactants in cellulose nanowhisker/epoxy nanocomposites: effect on filler dispersion and system properties, Cellulose. 22 (2015) 3161-3176. doi:10.1007/s10570-015-0728-6.

[47] H.A. Al-Turaif, Relationship between tensile properties and film formation kinetics of epoxy resin reinforced with nanofibrillated cellulose, Prog. Org. Coatings. 76 (2013) 477481. doi:10.1016/j.porgcoat.2012.11.001.

[48] S.X. Peng, H. Chang, S. Kumar, R.J. Moon, J.P. Youngblood, A comparative guide to controlled hydrophobization of cellulose nanocrystals via surface esterification, Cellulose. 23 (2016) 1825-1846. doi:10.1007/s10570-016-0912-3.

[49] Y. Yoo, J.P. Youngblood, Green One-Pot Synthesis of Surface Hydrophobized Cellulose Nanocrystals in Aqueous Medium, ACS Sustain. Chem. Eng. 4 (2016) 3927-3938. 
doi:10.1021/acssuschemeng.6b00781.

[50] R. Mezzenga, L. Boogh, J.-A.E. Månson, Evaluation of solubility parameters during polymerisation of amine-cured epoxy resins, J. Polym. Sci. Part B Polym. Phys. 38 (2000) 1883-1892. doi:10.1002/1099-0488(20000715)38:14<1883::AID-POLB70>3.0.CO;2-M.

[51] N. Lin, J. Huang, P.R. Chang, J. Feng, J. Yu, Surface acetylation of cellulose nanocrystal and its reinforcing function in poly(lactic acid), Carbohydr. Polym. 83 (2011) 1834-1842. doi:10.1016/j.carbpol.2010.10.047.

[52] X. Zhang, P. Ma, Y. Zhang, Structure and properties of surface-acetylated cellulose nanocrystal/poly(butylene adipate-co-terephthalate) composites, Polym. Bull. (2016). doi:10.1007/s00289-015-1594-y.

[53] Z. Guo, T. Pereira, O. Choi, Y. Wang, H.T. Hahn, Surface functionalized alumina nanoparticle filled polymeric nanocomposites with enhanced mechanical properties, J. Mater. Chem. 16 (2006) 2800. doi:10.1039/b603020c.

[54] J.H. Zhu, S.Y. Wei, J. Ryu, M. Budhathoki, G. Liang, Z.H. Guo, In situ stabilized carbon nanofiber (CNF) reinforced epoxy nanocomposites, J. Mater. Chem. 20 (2010) $4937-$ 4948. doi:Doi 10.1039/C0jm00063a.

[55] J. Štěpek, H. Daoust, Additives for Plastics, Springer New York, New York, NY, 1983. doi:10.1007/978-1-4419-8481-4. 
Surface modified Cellulose Nanocrystals

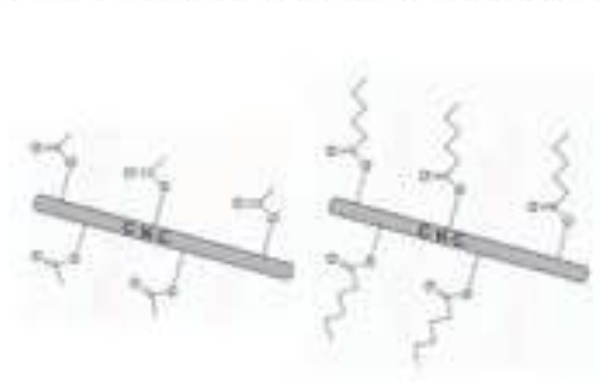

CNC_a
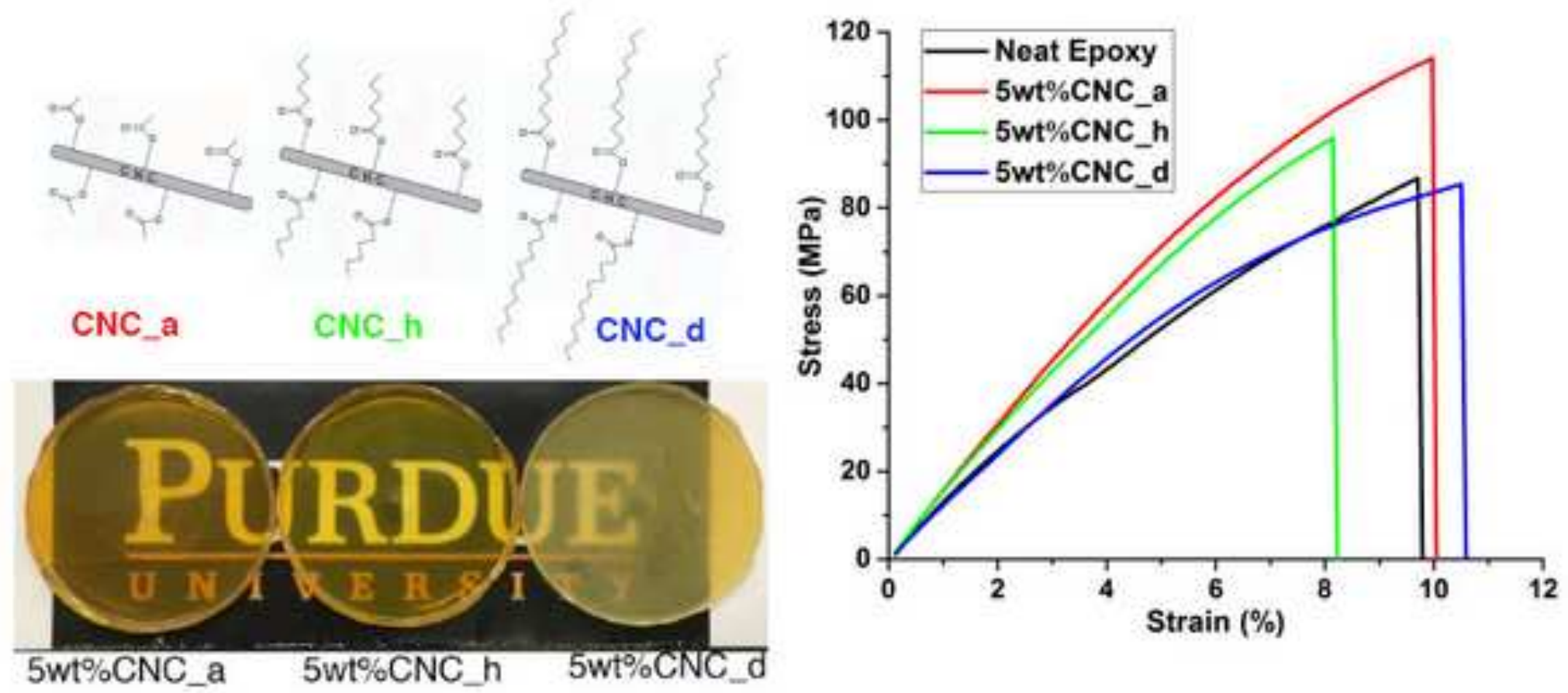